\title{
A phase II clinical trial of the Aurora and angiogenic kinase inhibitor ENMD-2076 for previously treated, advanced, or metastatic triple-negative breast cancer
}

Jennifer R. Diamond ${ }^{1,4^{*}}$ D, S. G. Eckhardt' ${ }^{2}$ Todd M. Pitts $^{1}$, Adrie van Bokhoven ${ }^{1}$, Dara Aisner ${ }^{1}$, Daniel L. Gustafson ${ }^{1}$, Anna Capasso², Sharon Sams ${ }^{1}$, Peter Kabos ${ }^{1}$, Kathryn Zolman', Tiffany Colvin ${ }^{1}$, Anthony D. Elias', Anna M. Storniolo ${ }^{3}$, Bryan P. Schneider ${ }^{3}$, Dexiang Gao ${ }^{1}$, John J. Tentler ${ }^{1}$, Virginia F. Borges ${ }^{1}$ and Kathy D. Miller ${ }^{3}$

\begin{abstract}
Background: Triple-negative breast cancer (TNBC) remains an aggressive breast cancer subtype with limited treatment options. ENMD-2076 is a small-molecule inhibitor of Aurora and angiogenic kinases with proapoptotic and antiproliferative activity in preclinical models of TNBC.

Methods: This dual-institution, single-arm, two-stage, phase II clinical trial enrolled patients with locally advanced or metastatic TNBC previously treated with one to three prior lines of chemotherapy in the advanced setting. Patients were treated with ENMD-2076 250 mg orally once daily with continuous dosing in 4-week cycles until disease progression or unacceptable toxicity occurred. The primary endpoint was 6-month clinical benefit rate (CBR), and secondary endpoints included progression-free survival, pharmacokinetic profile, safety, and biologic correlates in archival and fresh serial tumor biopsies in a subset of patients.
\end{abstract}

Results: Forty-one patients were enrolled. The 6-month CBR was $16.7 \%(95 \% \mathrm{Cl}, 6-32.8 \%)$ and included two partial responses. The 4-month CBR was $27.8 \%$ (95\% Cl, 14-45.2\%), and the average duration of benefit was 6.5 cycles. Common adverse events included hypertension, fatigue, diarrhea, and nausea. Treatment with ENMD-2076 resulted in a decrease in cellular proliferation and microvessel density and an increase in p53 and p73 expression, consistent with preclinical observations.

Conclusions: Single-agent ENMD-2076 treatment resulted in partial response or clinical benefit lasting more than 6 months in $16.7 \%$ of patients with pretreated, advanced, or metastatic TNBC. These results support the development of predictive biomarkers using archival and fresh tumor tissue, as well as consideration of mechanism-based combination strategies.

Trial registration: ClinicalTrials.gov, NCT01639248. Registered on July 12, 2012.

Keywords: Breast cancer, ENMD-2076, Aurora kinase inhibitor, Triple negative

\footnotetext{
*Correspondence: jennifer.diamond@ucdenver.edu

${ }^{1}$ University of Colorado Cancer Center, Aurora, CO, USA

${ }^{4}$ Division of Medical Oncology, University of Colorado Anschutz Medical

Campus, University of Colorado Cancer Center, 12801 East 17th Avenue,

Mailstop 8117, Aurora, CO 80045, USA

Full list of author information is available at the end of the article
}

(c) The Author(s). 2018 Open Access This article is distributed under the terms of the Creative Commons Attribution 4.0 International License (http://creativecommons.org/licenses/by/4.0/), which permits unrestricted use, distribution, and reproduction in any medium, provided you give appropriate credit to the original author(s) and the source, provide a link to the Creative Commons license, and indicate if changes were made. The Creative Commons Public Domain Dedication waiver (http://creativecommons.org/publicdomain/zero/1.0/) applies to the data made available in this article, unless otherwise stated. 


\section{Background}

Triple-negative breast cancer (TNBC) is an aggressive breast cancer subtype defined by a lack of expression of the estrogen receptor (ER), progesterone receptor, and human epidermal growth factor receptor 2 (HER2) [1]. TNBC accounts for $10-15 \%$ of all newly diagnosed breast cancer and is associated with an increased risk of distant metastasis and death compared with other breast cancer subtypes [1-3]. The median duration of first-line therapy for metastatic TNBC is approximately 12 weeks, and the median survival for patients with metastatic disease is $10-13$ months $[2,4]$. Despite the characterization of biologic subtypes within TNBC, this disease remains critically in need of effective targeted systemic therapies [5].

ENMD-2076 is an orally bioavailable small-molecule inhibitor of angiogenic and mitotic kinases. The antiangiogenic activity of ENMD-2076 is mediated through the inhibition of vascular endothelial growth factor receptors (VEGFRs) and fibroblast growth factor receptors (FGFRs), whereas antiproliferative activity occurs via inhibition of mitotic kinases, including Aurora kinase A (Aur A) [6]. ENMD-2076 is active against preclinical TNBC models, including p53-mutated cancer cell lines and patient-derived tumor xenograft (PDX) models [7, 8]. The purpose of this study was to evaluate the anticancer activity of ENMD-2076 in patients with previously treated locally advanced or metastatic TNBC, to further characterize the side effect profile, and to explore pharmacodynamic changes in serial tumor biopsies.

\section{Methods}

\section{Study design}

This phase II clinical trial was a dual-institution, single-arm, Simon two-stage study of single-agent ENMD-2076 administered orally once daily with continuous dosing (ClinicalTrials.gov identifier NCT01639248). The primary objective of the study was to determine the 6-month clinical benefit rate (CBR), defined as patients with complete response, partial response (PR), or stable disease (SD) lasting for $\geq$ 24 weeks based on Response Evaluation Criteria in Solid Tumors (RECIST version 1.1) [9]. Secondary objectives included progression-free survival (PFS), objective response rate, safety, and pharmacokinetics (PK). Exploratory objectives included evaluation of p53 mutation status on archival tumor tissue and pharmacodynamic effects of ENMD-2076 in serial tumor tissue samples obtained in a subset of patients. This protocol was approved by the institutional review boards of both institutions, and informed consent was obtained from all patients prior to performing study-related procedures in accordance with federal and institutional guidelines.

\section{Eligibility criteria}

Eligible patients had locally advanced or metastatic TNBC previously treated with one to three prior lines of chemotherapy in the advanced setting. For the purpose of this study, locally advanced breast cancer was defined as unresectable local or regional disease. TNBC was defined as negative for estrogen and progesterone receptor by local pathology report, Allred score $\leq 2$, or $<5 \%$ weak positive staining. Negative HER2 testing was defined as IHC score 0 or $1+$ or fluorescence in situ hybridization ratio $<2.0$. Patients were required to have measurable disease by RECIST 1.1 [9], Eastern Cooperative Oncology Group (ECOG) Performance Status of 0-1, and archival tumor tissue available for analysis. Patients also had to be aged 18 years or older and to have adequate hematopoietic, hepatic, and kidney function, defined as hemoglobin $\geq 9 \mathrm{~g} / \mathrm{dl}$, absolute neutrophil count $\geq 1500 /$ $\mu \mathrm{l}$, platelets $\geq 100,000 / \mu \mathrm{l}$, total bilirubin $<1.5$ times the institutional upper limit of normal (ULN), aspartate aminotransferase/alanine aminotransferase and $\leq 2.5$ times the ULN or $<5$ times the ULN if hepatic metastases were present, and creatinine $\leq 1.5$ times the ULN. Brain magnetic resonance imaging (MRI) was required to exclude brain metastasis. For patients undergoing the optional serial tumor biopsies, prothrombin time and activated partial thromboplastin time were required to be within the normal range. Patients were recovered from the expected toxicity of prior treatments and did not require therapeutic doses of any anticoagulant or have any significant cardiac problems.

\section{Pretreatment evaluation}

Prior to the initiation of study treatment, all patients underwent clinical history and physical examination, ECOG Performance Status assessment, vital signs, complete blood count, chemistries, urinalysis, serum pregnancy test (if applicable), coagulation parameters (for patients undergoing tumor biopsies), echocardiogram or multigated acquisition, and baseline tumor assessment with imaging. Archival tissue was submitted for correlative analysis for all enrolled patients. Additionally, serial tumor biopsies were performed for correlative analysis in a subset of patients, with the first occurring before the initiation of study treatment.

\section{Treatment and dose modifications}

Patients were treated with ENMD-2076 $250 \mathrm{mg}$ oral once daily with continuous dosing in 4-week cycles until disease progression or unacceptable toxicity occurred. The dose of study drug was selected on the basis of tolerability of ENMD-2076 in a phase II clinical trial in patients with recurrent, platinum-resistant ovarian cancer where the initial ENMD-2076 dose was $325 \mathrm{mg}$ oral once daily for patients with a body surface area (BSA) $\geq$ $1.65 \mathrm{~m}^{2}$ and $275 \mathrm{mg} / \mathrm{d}$ for patients with a BSA $\leq 1.64 \mathrm{~m}^{2}$ [10]. Due to higher-than-expected rates of treatment-related toxicity resulting in dose delays in that 
study, the starting dose was reduced to $275 \mathrm{mg} / \mathrm{d}$ and $250 \mathrm{mg} / \mathrm{d}$ for the two BSA groups, respectively. Taking into account this tolerability data for ENMD-2076 administered on a once-daily continuous dosing schedule, a flat dose of $250 \mathrm{mg} / \mathrm{d}$ was selected as the starting dose for this study.

Restaging was performed every two cycles (8 weeks) according to RECIST 1.1. Blood pressure was monitored weekly during the first cycle, every 2 weeks during cycle 2 , and every 4 weeks during cycles 3 and beyond. Complete blood count and chemistries were performed every 2 weeks during cycles 1 and 2, then every 4 weeks. Urinalysis was performed every 4 weeks, and if $2+$ proteinuria was observed, a spot urine protein/creatinine ratio was calculated and a 24-hour urine sample was collected for quantification of protein. Patients were allowed to continue treatment if the spot urine/protein ratio was $\leq 1$ and the 24-hour urine showed $\leq 3.5 \mathrm{~g}$ protein $/ 24 \mathrm{~h}$. If the 24 -hour urine protein was $>3.5 \mathrm{~g}$, treatment was withheld until a repeat study was $\leq$ $3.5 \mathrm{~g}$ protein $/ 24 \mathrm{~h}$. Thyroid-stimulating hormone was tested on cycle 3 , day 1 and subsequently as clinically indicated.

Dosing delays of up to 2 weeks were permitted to allow for recovery from treatment-related toxicities or other intercurrent illness. Longer delays were also allowed in patients experiencing clinical benefit from ENMD-2076 treatment. Two dose reductions were allowed for treatment-related toxicity (dose -1 was $225 \mathrm{mg} / \mathrm{d}$ and dose -2 was $150 \mathrm{mg} / \mathrm{d}$ ). The protocol did not dictate specific management strategies for ENMD-2076-related treatment toxicities, including hypertension. The treatment management strategy was at the discretion of the treating physician.

\section{Pharmacokinetic sampling and assay}

Blood samples were collected in sodium heparin tubes prior to the first dose on cycle 1 , day 1 and then 4 hours following dosing. Samples were obtained prior to dosing on cycle 2, day 1 and on the day of tumor biopsy. Plasma concentrations of ENMD-2076 and its active metabolite, ENMD-2060, were determined using a validated LC-MS/MS method. The lower limits of quantification were $2.5 \mathrm{ng} / \mathrm{ml}$ for ENMD-2076 and $1 \mathrm{ng} / \mathrm{ml}$ for ENMD-2060.

\section{Correlative studies using archival tumor tissue and serial tumor biopsies}

Archival tumor tissue was requested from all eligible patients and subject to evaluation for p53 mutation and IHC expression. If multiple samples were available, the most recent tumor tissue was selected for testing unless it was quantitatively insufficient. Microdissection was performed using a stereotactic microscope with hematoxylin-stained slides, and DNA was isolated for sequencing of TP53 exons 5-8 and 10. Briefly, following microdissection, samples were purified using the QIAamp DSP FFPE Tissue Kit (Qiagen, Valencia, CA, USA). Exons 5-8 and 10 were PCR-amplified followed by Sanger sequencing using the Applied Biosystems BigDye system (Life Technologies, Carlsbad, CA, USA), and capillary electrophoresis was performed on an ABI 3500xL instrument (Applied Biosystems/Thermo Fisher Scientific, Foster City, CA, USA). Sequence analysis was performed using MutationSurveyor software (SoftGenetics, State College, PA, USA). TP53 alterations were assessed for pathogenicity by first determining whether they represented population polymorphisms using the Exome Aggregation Consortium database (exac.broadinstitute.org). Any alteration with a population minor allele frequency $>1 \%$ was excluded from further analysis. All remaining identified alterations were examined for pathogenicity on the basis of classification by the International Agency for Research on Cancer TP53 Database (p53.iarc.fr), and all alterations classified as "deleterious" by this database were considered mutations for the purposes of subsequent analysis.

Serial tumor biopsies were performed in a subset of patients during the first cycle prior to day 1 and at days 14-16. An additional biopsy was obtained at the time of progression in patients responding to treatment. Formalin-fixed, paraffin-embedded samples were analyzed by IHC for Ki-67 (30-9; Ventana Medical Systems, Inc., Tucson, AZ, USA), cleaved caspase 3 (Cell Signaling Technology, Danvers, MA, USA), p53 (Cell Marque, Rocklin, CA, USA), and CD34 (Abcam, Cambridge, MA, USA). Following antigen retrieval and primary antibody incubation, IHC stains were visualized with the UltraView Universal DAB Detection Kit (Ki-67 and p53; Ventana Medical Systems, Inc.) or ImmPRESS HRP Antirabbit IgG (CD34 and cleaved caspase 3; Vector Laboratories, Burlingame, CA, USA). IHC for Ki-67 was scored as follows: low proliferative index $\leq 15 \%$ positive cells, intermediate $16-30 \%$, and high $>30 \%$. IHC for $\mathrm{p} 53$ was scored as low $\leq 15 \%$ positive cells, intermediate $16-30 \%$, and high $>30 \%$. The average number of vessels from three $\times 20$ magnification fields was used as the microvessel density (MVD) score. The cleaved caspase 3 score was determined by evaluating the percentage of stained tumor cells.

Immunofluorescence (IF) was performed in a subset of biopsy samples for 4',6-diamidino-2-phenylindole (DAPI), p53, p73, and BAX as previously described [8]. In brief, samples were freshly collected, placed into individual cryomolds (Sakura Finetek, Torrance, CA, USA) and embedded in Tissue-Tek optimum cutting compound (Sakura Finetek). Cryomold blocks were frozen in liquid nitrogen and individually cut into 5- $\mu \mathrm{m}$-thick 
sections by the University of Colorado Cancer Center Tissue Biobanking and Histology Shared Resource. Slides were fixed in a 1:1 ratio in a solution of methanol and acetone at $-20{ }^{\circ} \mathrm{C}$ for 10 minutes, blocked, and incubated with primary antibodies (p53, Cell Signaling Technology; p73, Abcam). Slides were washed three times in $1 \times \mathrm{PBS}$ and incubated with secondary antibodies (Alexa Fluor 555 or Alexa Fluor 488; Life Technologies) and counterstained with $300 \mathrm{nM}$ DAPI. Slides were mounted, and images were acquired using the FLUOVIEW FV1000 confocal microscope (Olympus America, Center Valley, PA, USA) at $\times 60$ magnification.

\section{Statistical methods}

The primary endpoint of the study was the 6-month CBR, defined as the sum of patients who experienced complete response, PR, and/or stable disease (SD) for $\geq 24$ weeks by RECIST version 1.1. The sample size was determined using a null hypothesis of $10 \%$ [11, 12] and an alternate hypothesis of interest to continue single-agent studies in the patient population of $30 \%$. The sample size of 35 yielded a power of $90 \%$ to detect this difference with an alpha of 0.05 (one-sided). Patients were considered evaluable for the primary endpoint if they received at least one cycle of therapy and underwent repeat disease assessment. $\mathrm{Pa}-$ tients who came off study during the first cycle for toxicity or patient preference without disease progression were not considered evaluable for the primary endpoint. This study used a two-stage study design. The interim analysis (stage 1) was performed after enrollment of 18 patients. If 2 or fewer of the first 18 patients experienced a "success", then it would be concluded that the treatment does not have sufficient activity for further investigation, and accrual would be terminated. If 3 or more of these 18 patients experienced a "success," enrollment would continue to 17 additional evaluable patients.

Descriptive statistics were used for PK and toxicity parameters. Fisher's exact test was used to assess the correlation between p53 mutation and response to treatment.

\section{Results \\ Patients}

Between July 2012 and October 2016, 65 patients were consented, and 41 patients were enrolled at the University of Colorado Cancer Center (Aurora, CO, USA) and the Indiana University Melvin and Bren Simon Cancer Center (Indianapolis, IN, USA). This included 18 patients enrolled in stage 1 and 23 patients in stage 2 (Fig. 1). A total of 24 patients were consented and excluded prior to starting treatment, most commonly for not meeting the eligibility criteria owing to asymptomatic brain metastasis detected on required screening brain MRI or abnormal liver function tests.

The median age of patients was 54 years (range, 3073 years); 40 were female, and 1 was male. Patients received, on average, 1.7 prior lines of chemotherapy for locally advanced unresectable or metastatic disease, and $80.5 \%$ underwent prior neoadjuvant or adjuvant chemotherapy for localized disease. In addition, $48.8 \%$ of patients received prior systemic targeted anticancer therapies, and $85.4 \%$ received prior radiation therapy. ECOG Performance Status was 1 in $53.7 \%$ of patients and 0 in $46.3 \%$ of patients. BRCA1/2 germline mutation status was collected if known; 9.8\% had known

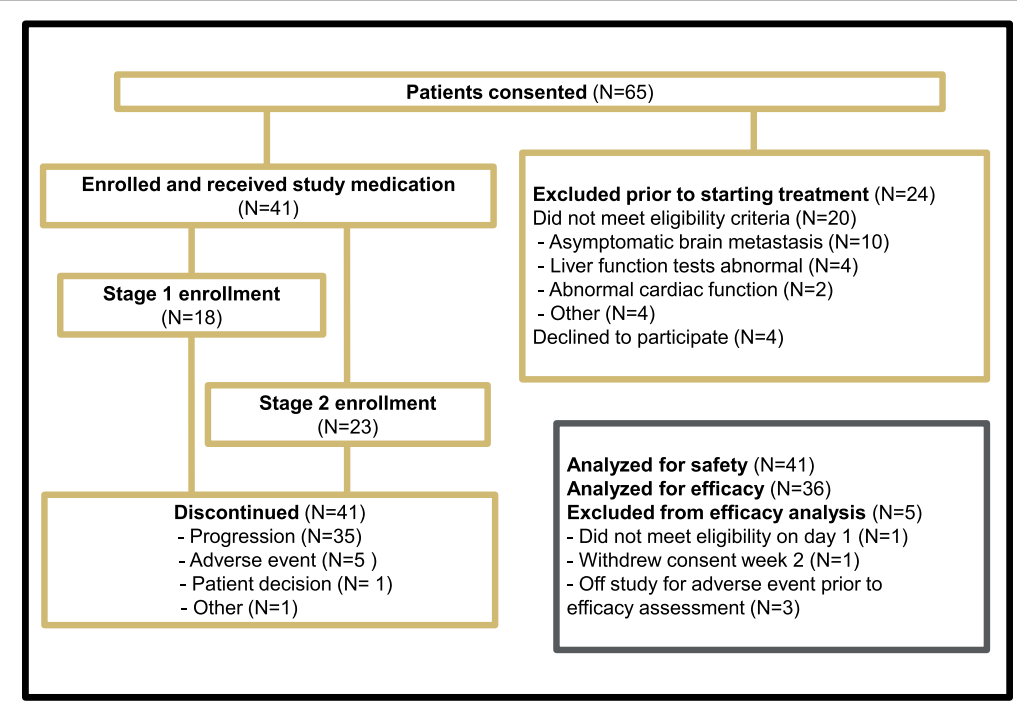

Fig. 1 Trial profile and study design 
deleterious mutations, $46.3 \%$ were known wild type, and status was unknown in $43.9 \%$ of patients. Additional baseline patient characteristics and demographics can be found in Table 1 .

\section{Efficacy}

Thirty-six patients were evaluable per protocol for the primary efficacy analysis. Five patients $(12.2 \%)$ were not included in the efficacy analysis owing to adverse events leading to discontinuation prior to objective efficacy assessment $(n=3)$, not meeting eligibility criteria on day $1(n=1)$, and withdrawal of consent in cycle $1(n=1)$ (Fig. 1). The study proceeded to the second stage of enrollment based on observing three 6-month CBR events in stage 1 ( $n=18$ patients). The 6-month $\mathrm{CBR}$ in the overall trial was $16.7 \%$ (exact 95\% CI, 6-32.8\%), and the 4-month CBR was $27.8 \%$ (exact 95\% CI, 14-45.2\%) (Table 2). Two patients achieved partial response to treatment, and no complete responses were observed. The average duration of response for patients achieving clinical benefit for 4 months was 6.5 cycles $(n=10)$ (Fig. 2). The median PFS for all patients treated with ENMD-2076 $(n=41)$ was 1.84 months (95\% CI, 1.81-3.68) (Fig. 2). The median PFS for all patients evaluable for the efficacy analysis $(n=36)$ was 1.86 months (95\% CI, 1.73-3.73).

\section{Safety profile}

The most common treatment-related adverse events were hypertension (66\%), fatigue (54\%), diarrhea (54\%), and nausea (49\%). Table 3 lists all treatment-related adverse events occurring in $10 \%$ or more of patients. Dose reduction occurred in eight patients $(20 \%)$ for fatigue, hypertension, and proteinuria. The most common grade 3 treatment-related adverse events were hypertension (37.5\%) and fatigue (10\%). One patient experienced grade 4 hypertension. Five patients (12.2\%) discontinued treatment owing to a treatment-related adverse event (Fig. 1).

\section{Pharmacokinetics}

The mean steady-state plasma concentration of ENMD-2076 at cycle 2 , day 1 was $441 \pm 394 \mathrm{ng} / \mathrm{ml}$. The mean steady-state concentration of its active metabolite, ENMD-2060, was $43.3 \pm 10.3 \mathrm{ng} / \mathrm{ml}$. The mean ratio of ENMD-2076 to ENMD-2060 was $10.3 \pm$ 4.9 .

Additional PK sampling was performed in patients undergoing serial tumor biopsies on the day of the biopsy. This was performed at cycle 1 , days 14-16. PK sampling data was available for ten subjects undergoing serial tumor biopsy, and the mean plasma concentrations of ENMD-2076 and ENMD-2060 were $441 \pm$ $275 \mathrm{ng} / \mathrm{ml}$ and $40.1 \pm 22.9 \mathrm{ng} / \mathrm{ml}$, respectively.

\section{Correlative analysis of serial tumor biopsies and archival tumor tissue}

A total of 15 patients underwent at least one tumor biopsy analysis. Eight patients had predose and postdose samples (days 14-16) with sufficient tissue for IHC analysis. One patient underwent an additional biopsy at the time of disease progression following treatment for ten cycles.

Analysis of serial tumor biopsies prior to and following 2 weeks of ENMD-2076 ( $n=8$ patients) demonstrated a treatment-induced decrease in cellular proliferation (Ki-67) (Fig. 3a) and MVD (CD34) (Fig. 3b) as assessed by IHC. This was a trend observed in patients regardless of tumor response to treatment by RECIST 1.1. An increase in cleaved caspase 3 as a marker of apoptosis was only observed in association with response to treatment and not in nonresponders (Figs. 3c and d). The posttreatment decrease in $\mathrm{Ki}-67$ and increase in cleaved caspase 3 observed in patient 01-028 (Fig. 3c, responder) was lost at the time of progression at day 280 of treatment, consistent with preclinical modeling of ENMD-2076 activity in TNBC PDX models [8]. An increase in MVD was not observed at the time of acquired progression in this patient's tumor sample.

On the basis of emerging preclinical data, the final two patients undergoing serial tumor biopsies had their samples processed for IF to investigate changes in p53, p21, p73, BAX, and senescence-associated $\beta$-galactosidase (SA- $\beta$-gal) following treatment [8]. One patient had sufficient tissue obtained from the pretreatment and posttreatment samples for IF and SA- $\beta$-gal. In this patient, an increase in p53 and p73 expression was observed (Fig. 3e), in combination with an increase in SA- $\beta$-gal staining. DNA mutational testing of p53 revealed the presence of an R273S mutation in this patient's tumor, and the patient had SD for three cycles on study followed by progression. An increase in p53 family member expression following treatment is consistent with changes observed in preclinical TNBC PDX models treated with ENMD-2076 and other Aurora kinase inhibitors $[8,13]$.

Archival tumor tissue was obtained from all enrolled patients, and p53 mutation testing revealed deleterious missense mutations in $38.9 \%$, frameshift mutations in $13.9 \%$, wild type in $36.1 \%$, and status unknown for $11.1 \%$ (Fig. 3f). Of the six patients who had PR or SD for $>6$ months, $83 \%$ had a deleterious mutation in p53, as compared with $46 \%$ of those who did not meet that efficacy endpoint $(p=0.18)$. Archival tumor tissue samples were also subjected to p53 IHC. High p53 expression was defined as $>30 \%$ of cells expressing p53. High p53 expression was present in $16(50 \%)$ patients who had tissue available for analysis $(n=32)$, and high p53 expression plus p53 mutation was present in 11 of 32 (34.4\%). High 
Table 1 Baseline demographics and patient characteristics

Characteristic
Age, years
Median (range)
Sex
Male
Female
Race
White
Black or African American
Unknown
Ethnicity
Hispanic or Latino
Not Hispanic or Latino
Unknown
ECOG Performance Status
0
1
Prior lines of systemic therapy
(locally advanced unresectable or metastatic disease)
Mean
No
Prior

Prior targeted systemic anticancer therapy

Yes

No

Prior radiation therapy

Yes

No

BRCA1/2 germline deleterious mutation status

$\begin{array}{ll}\text { Mutated } & 4(9.8 \%) \\ \text { Wildtype } & 19(46.3 \%) \\ \text { Unknown } & 18(43.9 \%)\end{array}$

Number of metastatic sites

1

2

$\geq 3$

Sites of metastasis

Lung

Lymph nodes

Liver
Number

of patients

$(N=41)$

$54(30-73)$

$1(2.4 \%)$

$40(97.6 \%)$

$33(80.5 \%)$

5 (12.2\%)

3 (7.3\%)

$6(14.6 \%)$

$30(73.2 \%)$

5 (12.2\%)

19 (46.3\%)

22 (53.7\%)

1.7

$21(51.2 \%)$

10 (24.4\%)

$10(24.4 \%)$

33 (80.5\%)

8 (19.5\%)

$20(48.8 \%)$

$21(51.2 \%)$

35 (85.4\%)

$6(14.6 \%)$

16 (39.0\%)

14 (34.1\%)

$11(26.8 \%)$

15 (24.4\%)

20 (48.8\%)

15 (24.4\%)
Table 1 Baseline demographics and patient characteristics (Continued)

\begin{tabular}{ll}
\hline Characteristic & $\begin{array}{l}\text { Number } \\
\text { of patients } \\
(N=41)\end{array}$ \\
\hline Bone & $16(39.0 \%)$ \\
Chest wall & $8(19.5 \%)$ \\
Other & $6(14.6 \%)$ \\
\hline ECOG Eastern Cooperative Oncology Group &
\end{tabular}

p53 expression was observed in 50\% of patients meeting the 6-month CBR endpoint and those not meeting this efficacy endpoint, showing no correlation between high expression of p53 by IHC and efficacy.

\section{Discussion}

This study demonstrates that ENMD-2076 has durable clinical activity in a subset of patients with previously treated metastatic TNBC. We observed $27.8 \%$ and $16.7 \%$ of patients achieving prolonged clinical benefit at 4 and 6 months, respectively. The average duration of response for patients achieving clinical benefit for 4 months was 6.5 cycles. The prolonged benefit observed in a subset of patients is meaningful, given the aggressive nature of TNBC and low response rates to single-agent chemotherapies used in clinical practice. In a retrospective multicenter review of 111 patients with metastatic TNBC treated with standard-of-care chemotherapy, Kassam et al. found the median duration of therapy to be 9 weeks in the second-line setting and just 4 weeks in the third-line setting. Only $50 \%$ of patients went on to receive third-line therapy [4]. In a more recent analysis of the comparative effectiveness of eribulin, capecitabine, gemcitabine, and navelbine in metastatic previously treated TNBC, Dranitsaris et al. reported a median duration of therapy of 1.6-2 months for these agents [14]. There remains a critical unmet need for effective, targeted therapies to treat TNBC, and to achieve this will likely require patient selection strategies to identify a responding population for new therapies.

ENMD-2076 treatment resulted in mechanism-based toxicities, most commonly hypertension, fatigue, and diarrhea, consistent with prior clinical trials in solid

Table 2 Efficacy analysis

\begin{tabular}{ll}
\hline Efficacy response & $\begin{array}{l}\text { Number of patients } \\
(N=36)\end{array}$ \\
\hline Complete response & $0(0 \%)$ \\
Partial response & $2(5.6 \%)$ \\
Stable disease & $14(38.9 \%)$ \\
Progressive disease & $20(55.6 \%)$ \\
4-Month clinical benefit rate (4-CBR) & $10(27.8 \%)$ \\
6-Month clinical benefit rate (6-CBR) & $6(16.7 \%)$ \\
\hline
\end{tabular}




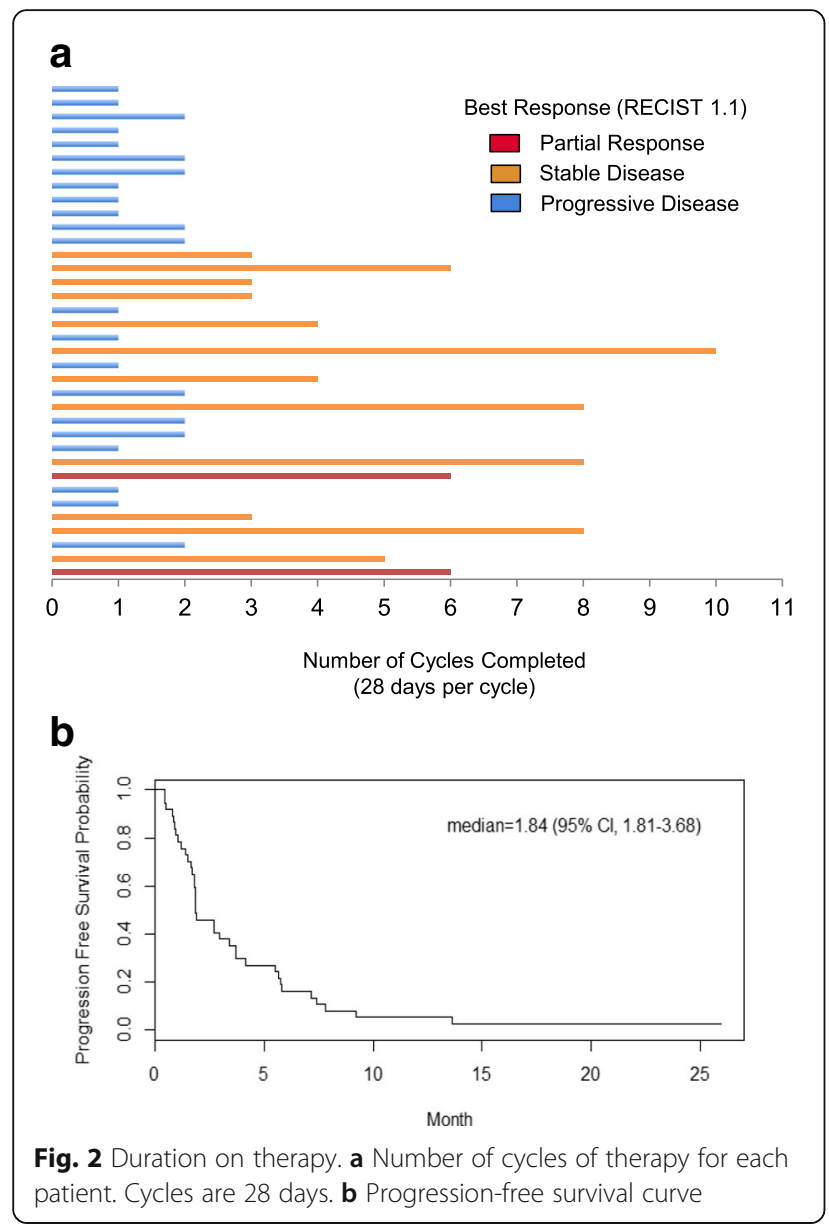

Table 3 Treatment-related adverse events occurring in 10\% or more of patients

\begin{tabular}{lll}
\hline Adverse event & No. of patients $(N=41)$ & \\
\cline { 2 - 3 } & All grades & Grade $\geq 3$ \\
\hline Hypertension & $27(66 \%)$ & $16(39 \%)$ \\
Fatigue & $22(54 \%)$ & $4(10 \%)$ \\
Diarrhea & $22(54 \%)$ & $1(2 \%)$ \\
Nausea & $20(49 \%)$ & $0(0 \%)$ \\
Constipation & $10(24 \%)$ & $0(0 \%)$ \\
Headaches & $10(24 \%)$ & $0(0 \%)$ \\
Vomiting & $9(22 \%)$ & $1(2 \%)$ \\
Mucositis & $6(15 \%)$ & $0(0 \%)$ \\
Proteinuria & $6(15 \%)$ & $3(7 \%)$ \\
Dysgeusia & $5(12 \%)$ & $0(0 \%)$ \\
GERD & $5(12 \%)$ & $0(0 \%)$ \\
Anorexia & $4(10 \%)$ & $0(0 \%)$ \\
\hline
\end{tabular}

GERD Gastroesophageal reflux disease tumor patients $[10,15]$. Hematologic toxicity was not common in this study. The majority of patients (66\%) experienced hypertension, and for $39 \%$ it was grade 3 or higher. This incidence and severity are similar to rates observed in prior studies of ENMD-2076 in patients with cancer and is likely related to VEGFR2 inhibition $[10,15]$. In most cases, hypertension was easily controlled with antihypertensive agents, most commonly a dihydropyridine calcium channel blocker or an angiotensin-converting enzyme inhibitor. Close monitoring of blood pressure and careful management of hypertension should continue to be required in future studies of ENMD-2076.

Despite lowering the starting dose in this trial from that used in the phase II ovarian cancer trial, dose reduction was required in $20 \%$ of patients, and $12.2 \%$ of patients discontinued treatment owing to treatment-related toxicity [10]. These rates of toxicity and dose modification are similar to those for other approved multitarget tyrosine kinase inhibitors, including sorafenib [16]. Limited PK analysis performed in this study demonstrated a mean steady-state plasma concentration of ENMD-2076 at cycle 2, day 1 of $441 \pm 394 \mathrm{ng} / \mathrm{ml}$, which is similar to $356 \mathrm{ng} / \mathrm{ml}$ observed in patients with ovarian cancer [10]. The toxicity observed in this trial should be considered in the design of future studies of ENMD-2076, and the incorporation of standard toxicity management algorithms may assist in the management of hypertension.

This trial incorporated archival tumor tissue analysis for p53 mutation and protein expression, as well as serial tumor biopsies in a subset of patients. These correlative studies were designed to further delineate the mechanism of action of this multitarget drug using human tumor samples and to explore a potential relationship between deleterious p53 mutations and ENMD-2076 activity in TNBC. Although these studies are exploratory in nature owing to a limited sample size, analysis can be performed in the context of previously published preclinical studies $[7,8,13]$.

As expected, ENMD-2076 was antiangiogenic, as evidenced by a decrease in blood vessel formation in tumor tissue following 2 weeks of treatment. ENMD-2076 is a potent inhibitor of the angiogenic kinases VEGFR2 (half-maximal inhibitory concentration $\left[\mathrm{IC}_{50}\right], 58 \mathrm{nM}$ ), VEGFR3 ( $\left.\mathrm{IC}_{50}, 16 \mathrm{nM}\right)$, FGFR1/2 ( $\mathrm{IC}_{50}, 71$ and $93 \mathrm{nM}$, respectively), and platelet-derived growth factor receptor- $\alpha\left(\mathrm{IC}_{50}, 56 \mathrm{nM}\right)$ [7]. We previously demonstrated in vivo that administration of ENMD-2076 treatment results in a decrease in MVD, as well as vascular permeability and perfusion, as measured by dynamic contrast-enhanced MRI [17]. TNBC commonly overexpresses vascular endothelial growth factor, which acts to promote angiogenesis and early metastatic potential 


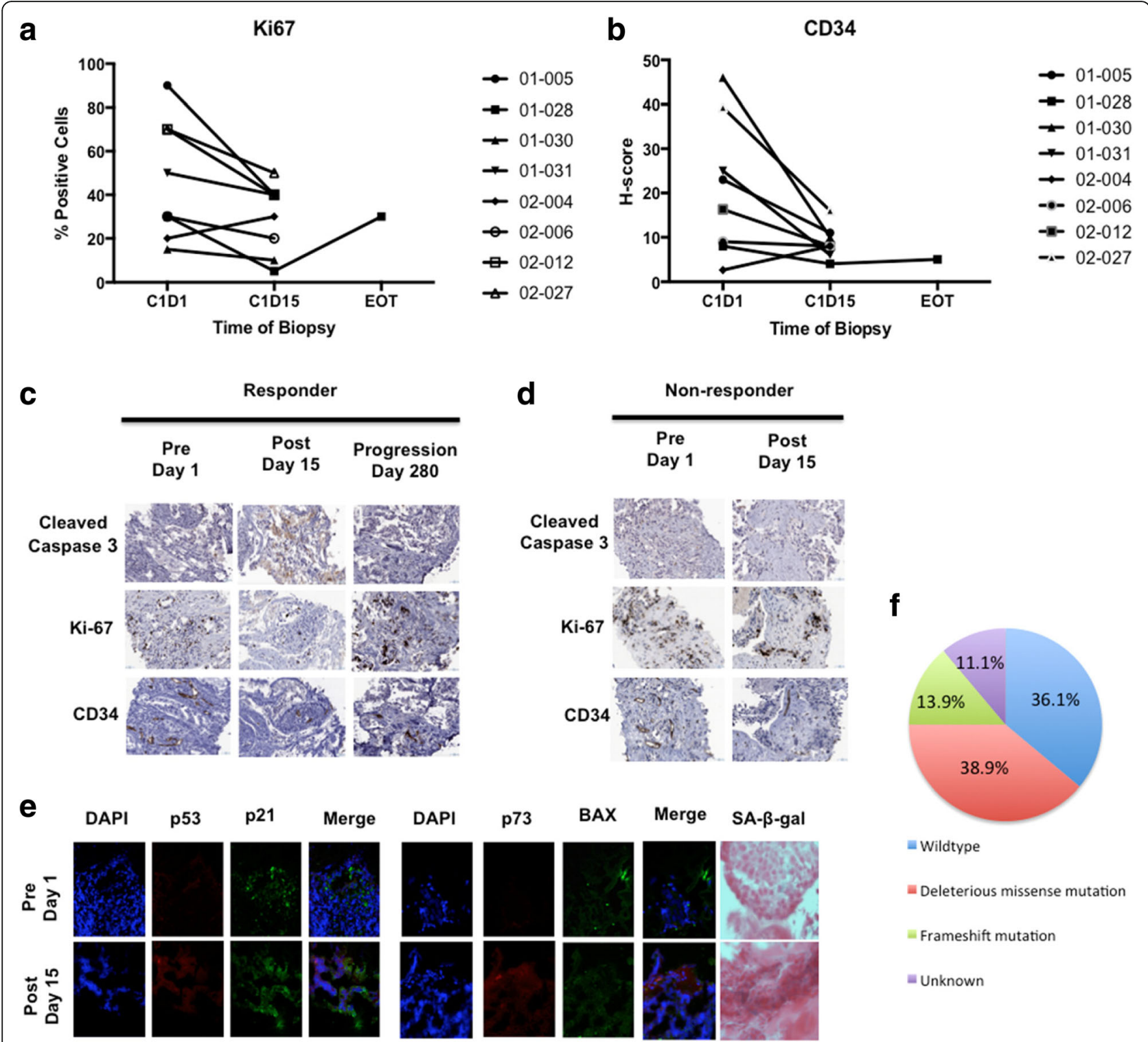

Fig. 3 Effects of ENMD-2076 on pharmacodynamic markers in serial tumor biopsies obtained in a subset of patients. a Paired samples were available for eight patients at baseline prior to dosing (C1D1) and postdose on days 14-16 (C1D15). An additional sample was obtained in one patient who experienced stable disease for ten cycles followed by progression (end of treatment [EOT]). Tissue was analyzed by IHC for Ki-67 as a marker of cellular proliferation. $\mathbf{b}$ Nonresponder. Staining was performed as in panel $\mathbf{a}$. Note that there is no decrease in proliferation or increase in apoptosis in the nonresponder following ENMD-2076 treatment. Samples were analyzed by IHC for CD34 expression as a marker of microvessel density. Changes were independent of tumor response and clinical benefit to ENMD-2076 treatment. Patients 01-005, 01-030, 02-004, 02-006, 02-012, and 02-027 had progressive disease (PD) at first imaging assessment following two cycles; 01-028 had stable disease (SD) for ten cycles; and 01-031 had SD for four cycles. c Immunoflurorescence analysis of tumor biopsies for 4',6-diamidino-2-phenylindole (DAPI), p53, and p73 in a patient who had stable disease by Response Evaluation Criteria in Solid Tumors (RECIST version 1.1) after two cycles of treatment and then progressed after cycle 3. Patient has a p53 mutation R273S. Note an increase in p53 and p73 following treatment, which is consistent with preclinical findings in patient-derived tumor xenograft models. IHC images from 01-028 responder. Cleaved caspase 3, Ki-67, and CD34 on serial tumor biopsies were used to assess apoptosis, proliferation, and microvessel density, respectively, in a patient responding to ENMD-2076 treatment with prolonged stable disease for ten cycles. Biopsies were obtained prior to treatment, 15 days after treatment, and at the time of disease progression day 280. Formalin-fixed, paraffin-embedded tissue sections were stained with the indicated antibodies, and representative images were obtained at $\times 20$ magnification. Note an increase in cleaved caspase 3 and a decrease in Ki-67 and CD34 in the posttreatment biopsy. At the time of disease progression, these changes were reversed. Changes in (d) Ki-67 and (e) CD34 (microvessel density) in serial tumor biopsies. Baseline and day 15 samples were available for eight patients. An additional sample was obtained from one patient at the time of progression following prolonged stable disease. Ki-67 and CD34 were assessed using IHC. SA- $\beta$-gal Senescence-associated $\beta$-galactosidase 
$[18,19]$. Similar to other trials, this study did not demonstrate a correlation between a decrease in MVD and clinical benefit. This highlights the multifaceted mechanisms of cancer progression and that inhibiting angiogenesis alone is unlikely to be sufficient to lead to durable tumor response in TNBC.

A decrease in cellular proliferation that was also independent of response to therapy was observed and is likely a result of Aurora kinase inhibition. ENMD-2076 is more selective for Aur A than Aurora kinase B ( $\mathrm{IC}_{50}$, $14 \mathrm{nM}$ and $350 \mathrm{nM}$, respectively); however, inhibition of both is likely in tumor tissue, based on the drug exposure observed in this study. The early time point selected for the on-treatment biopsy may have resulted in capturing a decrease in cellular proliferation that occurred early and was lost quickly with tumor progression at the 2-month repeat imaging time point. As expected, induction of apoptosis was observed only in association with a favorable response to treatment, consistent with preclinical models [13]. ENMD-2076 treatment-emergent increases in p53 and p73 expression were observed, also consistent with preclinical data.

We observed p53 mutations in the majority of patients, as expected on the basis of the known genomic landscape of TNBC [20]. Deleterious mutations in p53 were more common in patients with clinical benefit for $>6$ months; however, this was not statistically significant, owing to the small sample size. An increased sensitivity of p53-deficient models to Aurora kinase inhibitors, including AZD1152, AMG 900, and ENMD-2076, has been previously reported $[7,21,22]$. This is likely related to an impaired p53-dependent cell cycle checkpoint, which results in cells continuing to cycle through aberrant mitoses following drug exposure, ultimately resulting in cell death.

In this study, we observed a relatively high incidence of asymptomatic brain metastasis. Approximately 15\% (10 of 65 patients consented) of patients in this trial had asymptomatic brain metastasis detected on the required screening brain MRI. Patients with TNBC are more likely than patients with hormone receptor-positive, HER2-negative breast cancer to develop brain metastasis. Approximately $50 \%$ of patients with metastatic TNBC will develop brain metastasis over the course of their disease [23-26]. These patients were not eligible for enrollment in this trial, owing to concerns regarding the risk of hemorrhagic events at the time of trial conception; however, data are now available to support the safety of antiangiogenics in asymptomatic brain metastasis [27]. Patients with stable, asymptomatic brain metastasis previously treated with radiation therapy could be considered for future trials of ENMD-2076. The propensity for asymptomatic brain metastasis should be considered in the design of future metastatic TNBC trials.

\section{Conclusions}

ENMD-2076 has meaningful clinical benefit in a small subset of patients with previously treated metastatic TNBC. Future studies should continue to evaluate the relationship between p53 and response to treatment with a focus on the development of a patient selection strategy. Currently ongoing studies include a phase II clinical trial of ENMD-2076 in patients with metastatic previously treated TNBC in Asia, and biomarker development work is ongoing. This trial demonstrates the feasibility of performing serial tumor biopsies in multisite trials in metastatic TNBC and highlights the importance of these studies in confirming data generated in preclinical models. Combination therapies with immunotherapy and mammalian target of rapamycin pathway inhibitors are also under investigation based on ongoing preclinical work [8].

\section{Acknowledgements}

The University of Colorado Cancer Center Biobanking and Histology Shared Resource and Molecular Pathology Shared Resource were used for this project.

\section{Funding}

This work was supported by the National Institutes of Health $(\mathrm{NIH})$ and the National Cancer Institute (NCI) through grants 5P30CA046934-25 (University of Colorado Cancer Center Support Grant), 1R21CA164617-01A1 (to JRD), and 1K23CA172691-01A1 (to JRD). This funding was used for study design and collection, analysis and interpretation of data, and the writing of the manuscript. Support was also received from CASI Pharmaceuticals in the form of provision of study medication and coverage of some clinical care costs for enrolled patients.

Availability of data and materials

The datasets generated and/or analyzed during the current study are not publicly available, owing to restrictions in the informed consent document signed by the patients enrolled in this trial.

\section{Authors' contributions}

JRD led development of the concept and design of the trial, data acquisition, analysis, and manuscript writing. SGE contributed to trial design and data analysis. TMP and JJT designed the correlative study sample acquisition protocol and did biomarker data analysis. DA and AvB contributed to trial design and data analysis for molecular studies and performed microdissection, DNA isolation, and p53 sequencing. DLG contributed to trial design, data acquisition, and analysis for pharmacokinetic analysis. AC performed correlative studies on biopsy samples and analyzed data for correlative studies. SS performed IHC analysis and scoring and interpretation of data. PK, ADE, VFB, and KDM contributed to trial concept and design as well as data acquisition, patient enrollment, and interpretation of clinical trial data. KZ and TC contributed to the trial design, acquisition of data, and analysis of data. DG developed the statistical design of the study and performed the final statistical analysis. AMS and BPS contributed to acquisition of data, patient enrollment, and analysis/interpretation of clinical trial data. All authors read and approved the final manuscript.

Ethics approval and consent to participate

This trial was approved by the Colorado Multiple Institutional Review Board (COMIRB) prior to patient enrollment (COMIRB no. 11-0684).

\section{Competing interests}

Scientific consulting (to SGE) and research funding (to JRD) were provided by CASI Pharmaceuticals. 


\section{Publisher's Note}

Springer Nature remains neutral with regard to jurisdictional claims in published maps and institutional affiliations.

\section{Author details}

${ }^{1}$ University of Colorado Cancer Center, Aurora, CO, USA. ${ }^{2}$ Department of Oncology, University of Texas at Austin, Dell Medical School, Austin, TX, USA. ${ }^{3}$ Indiana University Melvin and Bren Simon Cancer Center, Indianapolis, IN, USA. ${ }^{4}$ Division of Medical Oncology, University of Colorado Anschutz Medical Campus, University of Colorado Cancer Center, 12801 East 17th Avenue, Mailstop 8117, Aurora, CO 80045, USA.

Received: 11 May 2018 Accepted: 3 July 2018

Published online: 02 August 2018

\section{References}

1. Carey L, Winer E, Viale G, Cameron D, Gianni L. Triple-negative breast cancer: disease entity or title of convenience? Nat Rev Clin Oncol. 2010;7: 683-92.

2. Anders CK, Carey LA. Biology, metastatic patterns, and treatment of patients with triple-negative breast cancer. Clin Breast Cancer. 2009; 9(Suppl 2):S73-81

3. Perou CM, Sorlie T, Eisen MB, van de Rijn M, Jeffrey SS, Rees CA, et al. Molecular portraits of human breast tumours. Nature. 2000:406:747-52.

4. Kassam F, Enright K, Dent R, Dranitsaris G, Myers J, Flynn C, et al. Survival outcomes for patients with metastatic triple-negative breast cancer: implications for clinical practice and trial design. Clin Breast Cancer. 2009;9: 29-33.

5. Lehmann BD, Bauer JA, Chen X, Sanders ME, Chakravarthy AB, Shyr Y, et al. Identification of human triple-negative breast cancer subtypes and preclinical models for selection of targeted therapies. J Clin Invest. 2011;121: 2750-67.

6. Fletcher GC, Brokx RD, Denny TA, Hembrough TA, Plum SM, Fogler WE, et al. ENMD-2076 is an orally active kinase inhibitor with antiangiogenic and antiproliferative mechanisms of action. Mol Cancer Ther. 2011;10:126-37.

7. Diamond JR, Eckhardt SG, Tan AC, Newton TP, Selby HM, Brunkow KL, et al. Predictive biomarkers of sensitivity to the Aurora and angiogenic kinase inhibitor ENMD-2076 in preclinical breast cancer models. Clin Cancer Res. 2013:19:291-303.

8. Ionkina AA, Tentler JJ, Kim J, Capasso A, Pitts TM, Ryall KA, et al. Efficacy and molecular mechanisms of differentiated response to the Aurora and angiogenic kinase inhibitor ENMD-2076 in preclinical models of p53mutated triple-negative breast cancer. Front Oncol. 2017;7:94.

9. Therasse P, Arbuck SG, Eisenhauer EA, Wanders J, Kaplan RS, Rubinstein L, et al. New guidelines to evaluate the response to treatment in solid tumors. J Natl Cancer Inst. 2000;92:205-16.

10. Matulonis UA, Lee J, Lasonde B, Tew WP, Yehwalashet A, Matei D, et al. ENMD-2076, an oral inhibitor of angiogenic and proliferation kinases, has activity in recurrent, platinum resistant ovarian cancer. Eur J Cancer. 2013;49: $121-31$.

11. Martín M. Platinum compounds in the treatment of advanced breast cancer. Clin Breast Cancer. 2001:2:190-208. discussion 209

12. Finn RS, Bengala C, Ibrahim N, Roche H, Sparano J, Strauss LC, et al. Dasatinib as a single agent in triple-negative breast cancer: results of an open-label phase 2 study. Clin Cancer Res. 2011;17:6905-13.

13. Tentler JJ, lonkina A, Tan AC, Newton TP, Pitts TM, Glogowska MJ, et al. p53 family members regulate phenotypic response to Aurora kinase A inhibition in triple-negative breast cancer. Mol Cancer Ther. 2015;14:1117-29.

14. Dranitsaris G, Gluck S, Faria C, Cox D, Rugo H. Comparative effectiveness analysis of monotherapy with cytotoxic agents in triple-negative metastatic breast cancer in a community setting. Clin Ther. 2015;37:134-44.

15. Diamond JR, Bastos BR, Hansen RJ, Gustafson DL, Eckhardt SG, Kwak EL, et al. Phase I safety, pharmacokinetic, and pharmacodynamic study of ENMD2076, a novel angiogenic and Aurora kinase inhibitor, in patients with advanced solid tumors. Clin Cancer Res. 2011;17:849-60.

16. Li Y, Gao ZH, Qu XJ. The adverse effects of sorafenib in patients with advanced cancers. Basic Clin Pharmacol Toxicol. 2015:116:216-21.

17. Tentler JJ, Bradshaw-Pierce EL, Serkova NJ, Hasebroock KM, Pitts TM, Diamond JR, et al. Assessment of the in vivo antitumor effects of ENMD2076, a novel multitargeted kinase inhibitor, against primary and cell line- derived human colorectal cancer xenograft models. Clin Cancer Res. 2010; 16:2989-98.

18. Greenberg S, Rugo HS. Triple-negative breast cancer: role of antiangiogenic agents. Cancer J. 2010;16:33-8.

19. Andre F, Job B, Dessen P, Tordai A, Michiels S, Liedtke C, et al. Molecular characterization of breast cancer with high-resolution oligonucleotide comparative genomic hybridization array. Clin Cancer Res. 2009;15:441-51.

20. Pareja F, Geyer FC, Marchio C, Burke KA, Weigelt B, Reis-Filho JS. Triplenegative breast cancer: the importance of molecular and histologic subtyping, and recognition of low-grade variants. NPJ Breast Cancer. 2016;2:16036.

21. Kalous O, Conklin D, Desai AJ, Dering J, Goldstein J, Ginther C, et al. AMG 900, pan-Aurora kinase inhibitor, preferentially inhibits the proliferation of breast cancer cell lines with dysfunctional p53. Breast Cancer Res Treat. 2013:141:397-408.

22. Tao Y, Zhang P, Girdler F, Frascogna V, Castedo M, Bourhis J, et al. Enhancement of radiation response in p53-deficient cancer cells by the Aurora-B kinase inhibitor AZD1152. Oncogene. 2008;27:3244-55.

23. Lin NU, Claus E, Sohl J, Razzak AR, Arnaout A, Winer EP. Sites of distant recurrence and clinical outcomes in patients with metastatic triple-negative breast cancer: high incidence of central nervous system metastases. Cancer. 2008:113:2638-45.

24. Matsuo S, Watanabe J, Mitsuya K, Hayashi N, Nakasu Y, Hayashi M. Brain metastasis in patients with metastatic breast cancer in the real world: a single-institution, retrospective review of 12-year follow-up. Breast Cancer Res Treat. 2017:162:169-79.

25. Molnar IA, Molnar BA, Vizkeleti L, Fekete K, Tamas J, Deak P, et al. Breast carcinoma subtypes show different patterns of metastatic behavior. Virchows Arch. 2017:470:275-83.

26. Saraf A, Grubb CS, Hwang ME, Tai CH, Wu CC, Jani A, et al. Breast cancer subtype and stage are prognostic of time from breast cancer diagnosis to brain metastasis development. J Neurooncol. 2017;134:453-63.

27. Besse B, Le Moulec S, Mazieres J, Senellart H, Barlesi F, Chouaid C, et al. Bevacizumab in patients with nonsquamous non-small cell lung cancer and asymptomatic, untreated brain metastases (BRAIN): a nonrandomized, phase II study. Clin Cancer Res. 2015;21:1896-903.

Ready to submit your research? Choose BMC and benefit from

- fast, convenient online submission

- thorough peer review by experienced researchers in your field

- rapid publication on acceptance

- support for research data, including large and complex data types

- gold Open Access which fosters wider collaboration and increased citations

- maximum visibility for your research: over $100 \mathrm{M}$ website views per year

At $\mathrm{BMC}$, research is always in progress.

Learn more biomedcentral.com/submissions 